This is an Accepted Manuscript of an article

published in Town Planning Review, Vol 86(2), January 2015

available online: http://online.liverpooluniversitypress.co.uk/toc/tpr/86/2

Beatrix Haselsberger

Conference report

The Evolution of Planning Thought (Lecture Series)

$19+21+23$ May 2014

The Vienna University of Technology, Austria, is celebrating its two hundredth anniversary in 2015. The Evolution of Planning Thought project forms part of these celebrations and is composed of three core elements: (1) a lecture series by leading academic planning pioneers together with, (2) an inter-generational dialogue including the Planning Thought Award (dedicated to early career planning scholars); and, (3) a book to be published in 2015. This report covers the lecture series in Vienna in May 2014 which assembled fifteen highly respected planners, who have influenced and shaped the field of planning over the last five plus decades to speak about and discuss their personal experiences of those times and to reflect on the development of the discipline of planning.

\title{
Now or never
}

The 2000s saw the retirement of the first generation of planning professors, the pioneers of planning, who led its formation into an intellectual field. Unfortunately, some of these planning pioneers have already died (such as Brian McLoughlin, Jeremy Alden, Seymour Mandelbaum and more recently also Peter Hall). But not all!

The Evolution of Planning Thought project was born out of the need to unpack the roots as well as the transformation of planning through oral histories. These oral histories were collected in the context of personal values and experiences as well as in relation to an ever changing world, from those individuals who have first-hand knowledge. By capturing the reflections of various first generation planners this project provides an essential understanding of what were and what are the most salient elements of the planning discipline, which should be valued and taken forward by current and future generations of planners. Placing oral histories into the centre of discussions allows the overall resonance of 
key planning ideas and major planning achievements to be understood better and, by doing so, builds up the basis for clarifying and shaping the legacy to be taken forward. But the current and next generation of planners have only a finite amount of time left to accumulate this kind of information. When this highly influential generation is gone, their knowledge will be lost.

The Evolution of Planning Thought project is composed of three core elements: (1) a Lecture Series organised at the Vienna University of Technology, Austria in May 2014; (2) an Intergenerational Dialogue including the Planning Thought Award (dedicated to early career planning scholars) and (3) a Book which will be published in 2015.

\section{A memorable moment: A piece of history written in Vienna}

The coming together of Louis Albrechts, Rachelle Alterman, Michael Batty, Andreas Faludi, John Forester, John Friedmann, Cliff Hague, Peter Hall (via Skype), Patsy Healey, Charles Hoch, Judith Innes, Klaus Kunzmann, Peter Marcuse, Barrie Needham and Gerhard Schimak in Vienna, Austria in May 2014 can certainly be called a memorable moment. All of them came to explain, with the help of their own intellectual biography, how and why they have developed theories and conceptual tools, how these conceptual tools shaped the development of practice, how these planners organised themselves increasingly at an international and global scale, and the conceptual, institutional and practical lacuna that remain to be filled. Some readers may wonder why exactly these planning pioneers have been selected. It is certainly true that there are many more out there, who have also influenced and shaped the field of planning over the last $5+$ decades in one way or another and in all of the different countries around the world. This group, however, has been selected because each individual within the group has distributed their knowledge regularly at international planning conferences or in English publication outlets. In other words, they have influenced - and still influence - many planners outside of their countries of origin. Also, I must admit, that it was this group of people which has influenced me the most, mainly through reading, in the frame of my planning education at the Vienna University of Technology, Austria.

The results from the Evolution of Planning Thought Lecture Series will lead to a book in which the essays and biographical experiences of the contributors will be recounted for all to see. I should mention two things at this point. First, Luigi Mazza is also a part of this book project, but due to health reasons was not able to participate in the Vienna Lecture Series. Second, Peter Hall has unfortunately passed away this summer (July $30^{\text {th }} 2014$ ). His book chapter is probably his last piece of writing and we will treat it as his legacy in our book. This 
underlines once more the finite amount of time we, the current generation of planners, have to learn from the oral histories of our planning pioneers.

This book is edited by Beatrix Haselsberger (Vienna University of Technology, Austria) and her co-editor Laura Saija (University of Catania, Italy). Therefore, in addition to the Lecture Series, which was open to the public, a set of other activities (book workshops, editorial meetings, inter-generational dialogues between young planners and students from Vienna and the planning pioneers, a world café) took place as part of the Vienna symposium in May 2014. Bringing together the planning pioneers and asking them to address different audiences (students, planning practitioners, lecture series participants, etc.) has been a challenge for both the organisers as well as the aging pioneers. The average age of all the planning pioneers is 74 years. The events in Vienna facilitated an open and transparent dialogue about where we are as planners, how we have arrived here and where we are going into the future as a community. To give the readers of Town Planning Review some insights about what participants have taken away from these dialogues a few selected delegates have been asked to reflect about their impressions.

\section{Voices from participants}

"The Evolution of Planning Thought Symposium week (May 2014) was extremely valuable in its abundance and honesty. There were reoccurring fundamental messages from the symposium that need to be acknowledged as key elements of an improved strategy to deal with the challenges we are facing. Firstly - as professionals we can never be simply observers. Critical ways of thinking and proactive approach are necessary in order to drive the evolution of planning forward. However it is important to remember that where there is an opportunity to have power and impact, there is also the inherent responsibility that needs to be taken with it. Secondly, planning as a profession serves the public and, as such, needs to be prepared not only to listen but also to hear the concerns coming from all levels within society. And last, but by no means least: Never try and separate theory and practice, for both of them are inseparable parts of a holistic approach to planning bound by past experiences and the lessons that have come from them."

Milena Aleksieva, Master student at the University of Portsmouth, UK

"If I were to pick the most interesting and inspiring moments during my studies, this particular week in May 2014 would definitely be on top. Do you know that feeling, when you read something interesting and you are not able to fully understand what it is meant? During this particular week in May 2014 I had the chance to catch a glimpse of the life and career of 15 
highly respected planners of the first generation, whose work shaped the field of planning as we know it today. This experience made me realize how their planning thought emerged and developed and what experiences underlie their planning perspective. It was as if those pages I read became alive, telling real stories, reflecting on the different aspects of planning in time and inviting me to ask questions in order to fully understand their messages." Pintilie Alexandra, Master student at the Department for Spatial Planning, Vienna University of Technology, Austria

"The inter-generational dialogue of the 'Evolution of planning thought' has been an illuminative program in which I learnt that it is not possible to address different problems with the same approach. Every location, every situation, each historical time has different challenges and requires different approaches. In our era the discipline is asking for a more holistic point of view concerning the complexity of the space in which we live. It is not only a physical space, delimited by fixed administrative boundaries that prevent an equal development of adjacent territories, but is a space characterized by relations. The following challenge is how to organise the forms of governance that can support this conception. In doing that innovation as a new way of thinking and addressing situations without leading on already used concepts of territory-making, is the key instrument. Finally I would like to highlight the message that planning is not about controlling the future but that it is about how we can make the future we desire. In doing that a creative approach is required. Creative minds generate innovative solutions."

Chiara Andreotta, PhD student at the Department for Spatial Planning, Vienna University of Technology, Austria

"The interactions with the pioneers of planning resulted in immense clarity of my thoughts, as I could compare and relate with the experiences in their lives. The valuable suggestions and comments we received, not only left us confident of tackling new global challenges, but also motivated us to take on complex problems of the future. Critically some planning pioneers also indicated that planners do make mistakes and these mistakes are sources of learning. Many a times, results of planning interventions cannot be predicted and have its cause and effects/uncertainties. It is time now to rethink on planning relationship in a multidisciplinary dimension. Planning thus needs to be innovative, democratic and adapting with the globalizing and rapidly changing world." Kirti Badhe, PhD student at the Department for Spatial Planning, Vienna University of Technology, Austria 
"The Evolution of Planning Thought Lecture Series was distinguished from other gatherings by its capacity to penetrate the past with his highly experienced scholars. All in all, this Lecture Series had refreshed my "thirst" to remain on path for there is a great past to recollect on and a challenging future to confront on."

Martin Duma-Menghires, master student at the Department for Spatial Planning, Vienna University of Technology, Austria

"There is no greater satisfaction that a student can take from the academic teaching than the experience of being part of something very practical but at the same time very theoretical that will shape in a very personal manner the trajectory of his/hers path. For this reason, I believe that my experience of the whole process that I took part of has not yet ended, rather is just the beginning of something else that will come. Probably the most important lesson that I have learned from the whole symposium is that there is no such thing of planning recipes that we can take and apply. Having all the pioneer planners in front of me and hearing their stories that were shaped based on their specific cultures, believes, and experiences helped me understand more how planning in general has its own different tools to use and that no 'right' method can be thought but rather theories or concepts of it that can or should be translated into decision. And that the key of a decision stands into how planners should anticipate the future rather than trying to control it." Iulia Arina Luca, master student at the Department for Spatial Planning, Vienna University of Technology, Austria

\section{What next?}

A few weeks after the Vienna Symposium, the Evolution of Planning Thought roundtable organised at the Association of European Schools of Planning (AESOP) 2014 conference in Utrecht, NL turned out to be a very enriching follow-up event. Those of you who have missed all the Evolution of Planning Thought events so far might want to join us at our Association of Collegiate Schools of Planning (ACSP) 2014 roundtable in Philadelphia or at our keynote panel discussion at the AESOP 2015 conference in Prague. All of these initiatives seek to, on the one hand, assure us that the embodied knowledge is made accessible to as many different generations from as many different planning cultures as possible. On the other hand, it ensures that the book's value goes beyond mere retrospective and provides a solid source of information for current and future generations of planners. The book is our contribution to further enrich the debate about the future of planning. 

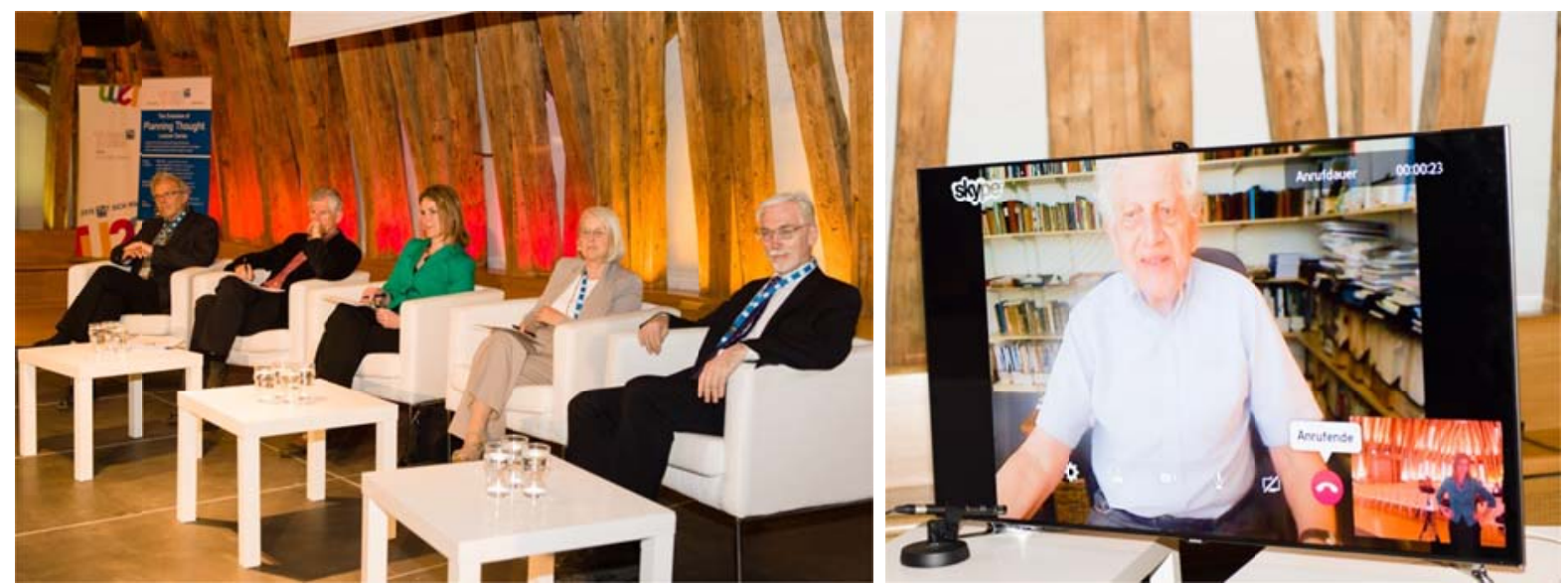

Left picture: Lecture 1: 19 May 2014. Andreas Faludi, Louis Albrechts, Beatrix Haselsberger, Judith Innes, Charles Hoch

Right picture: Lecture 1: 19 May 2014. Peter Hall participating in the Lecture via Skype
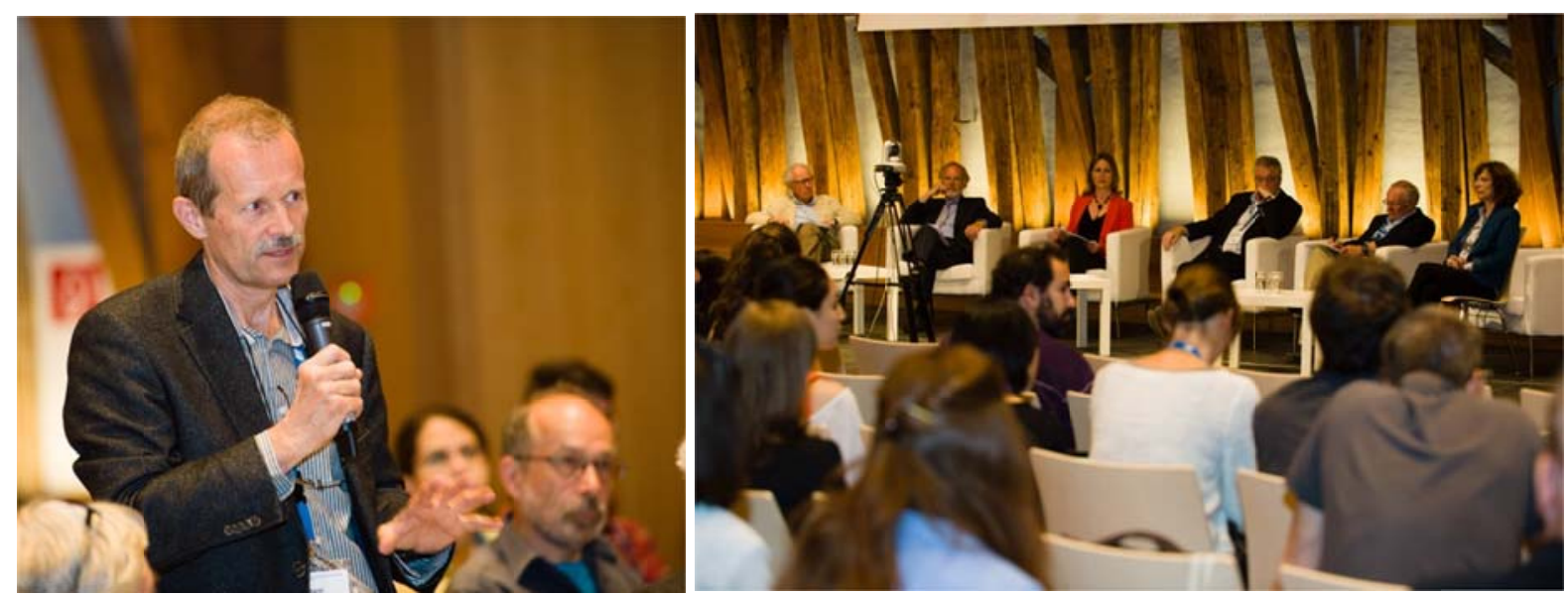

Left picture: Lecture 2: 21 May 2014. Karel Maier (participant) asking the panel for advice Right picture: Lecture 2: 21 May 2014. Peter Marcuse, Barrie Needham, Beatrix Haselsberger, Cliff Hague, Michael Batty, Rachelle Alterman
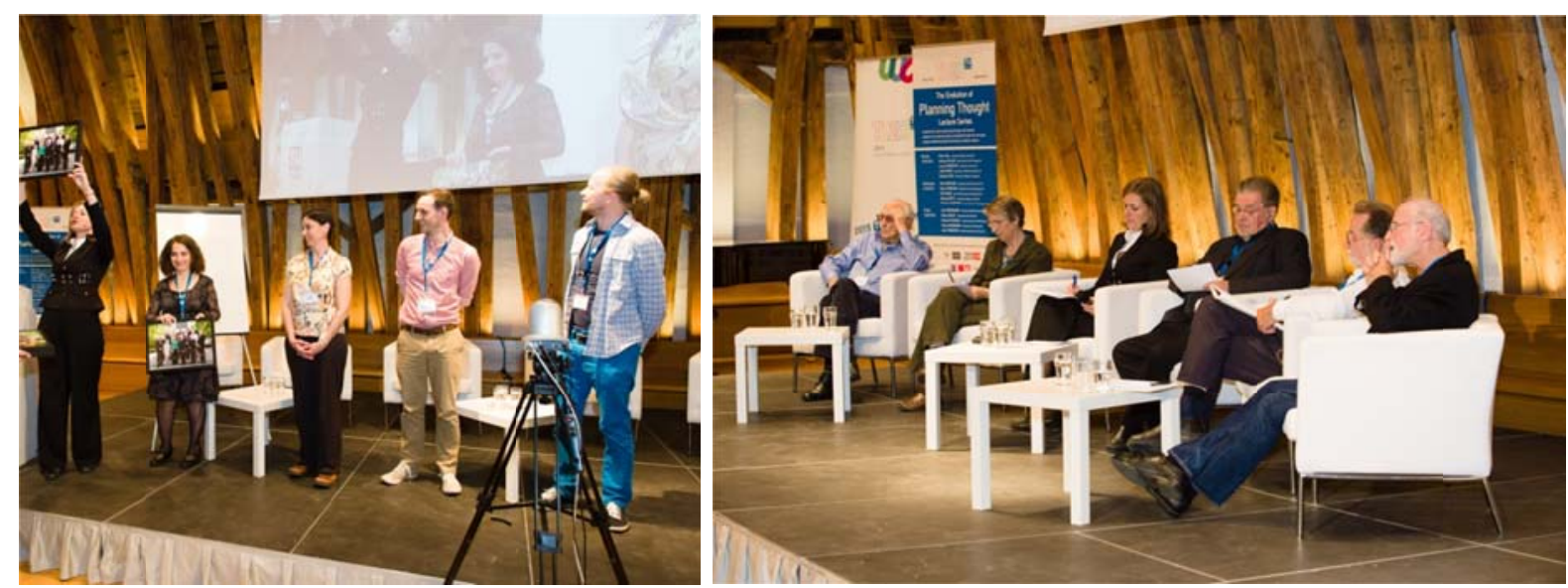

Left picture: The planning thought award winners: Sabrina Lai, Kathrine Quick, Chris Maidment, Juhu Matti Luukkonen

Right picture: Lecture 3: 23 May 2014. John Friedmann, Patsy Healey, Beatrix Haselsberger, Gerhard Schimak, Klaus Kunzmann, John Forester 


\section{Box 1:}

Further information about the Evolution of Planning Thought project is available at our website (http://info.tuwien.ac.at/planning-thought). As this book is designed for readers to tackle contemporary and future planning challenges and not for the bookshelf, we are eager to continue discussing these themes and therefore invite potential readers to get in touch with us via twitter (@PlanningThought).

\section{Box 2:}

Dr. Beatrix Haselsberger is a senior researcher at the Department of Spatial Planning of the Vienna University of Technology (Austria). In 2012, she was awarded the prestigious Hertha Firnberg Research Grant from the Austrian Science Fund for the project COMPASS (Collective Memory \& Planning: Across Social Separation), Project Number: T591-G16, which she is currently carrying out. The Evolution of Planning Thought book is one of the results out of this project. Contact: Beatrix.Haselsberger@tuwien.ac.at 